\title{
Assessment of phase-lag entropy, a new measure of electroencephalographic signals, for propofol-induced sedation
}

\author{
Mi Roung Jun ${ }^{1}$, Jae Hwa $\mathrm{Yoo}^{2}$, Sun Young Park ${ }^{2}$, Sojin $\mathrm{Na}^{2}$, Hyerim Kwon ${ }^{2}$, \\ Jae-Hwi Nho ${ }^{3}$, and Soon $\mathrm{Im} \mathrm{Kim}^{2}$ \\ ${ }^{I}$ Department of Anesthesiology and Pain Medicine, Samsung Changwon Hospital, Sungkyunkwan University School \\ of Medicine, Changwon, Departments of ${ }^{2}$ Anesthesiology and Pain Medicine, ${ }^{3}$ Orthopedic Surgery, Soonchunhyang \\ University Seoul Hospital, Seoul, Korea
}

\begin{abstract}
Background: Phase-lag entropy (PLE) was recently described as a measurement of temporal pattern diversity in the phase relationship between two electroencephalographic signals from prefrontal and frontal montages. This study was performed to evaluate the performance of PLE for assessing the depth of sedation.

Methods: Thirty adult patients undergoing upper limb surgery with a brachial plexus block were administered propofol by target-controlled infusion. The depth of sedation was assessed using the Observer's Assessment of Alertness/Sedation (OAA/S) scale. The effect-site concentration ( $\mathrm{Ce}$ ) of propofol was initially started at $0.5 \mu \mathrm{g} / \mathrm{ml}$ and was increased in increments of $0.2 \mu \mathrm{g} / \mathrm{ml}$ until an OAA/S score of 1 was reached. Three minutes after the target $C e$ was reached, the PLE, bispectral index (BIS), and level of sedation were assessed. Correlations between the OAA/S score and PLE or BIS were determined. The prediction probabilities $\left(\mathrm{P}_{\mathrm{k}}\right)$ of PLE and BIS were also analyzed.

Results: The PLE values were closely correlated with the OAA/S scores (Spearman's Rho $=0.755 ; \mathrm{P}<0.001$ ) to an extent comparable with the correlation between the BIS and OAA/S score (Spearman's Rho $=0.788 ; \mathrm{P}<0.001$ ). The $\mathrm{P}_{\mathrm{k}}$ values of PLE and BIS were 0.731 and 0.718 , respectively.

Conclusions: PLE is a new and reliable consciousness monitoring system for assessing the depth of sedation induced by propofol, which is comparable with the BIS.
\end{abstract}

Keywords: Consciousness monitor; Electroencephalography; Propofol; Sedation.

Corresponding author: Soon Im Kim, M.D., Ph.D.

Department of Anesthesiology and Pain Medicine, Soonchunhyang University Seoul Hospital, 59 Daesagwan-gil, Yongsan-gu, Seoul 04401, Korea

Tel: +82-2-709-9302, Fax: +82-2-790-0394

Email: anesikim@gmail.com

ORCID: https://orcid.org/0000-0001-8884-4096

This work was presented at ANESTHESIOLOGY 2018 Annual Meeting of The American Society of Anesthesiologists (ASA), October 2018, Moscone Center, San Francisco, CA, USA.

Received: January 16, 2019.

Revised: April 3, 2019 (1st); April 16, 2019 (2nd).

Accepted: April 16, 2019.

Korean J Anesthesiol 2019 August 72(4): 351-356

https://doi.org/10.4097/kja.d.19.00019

\section{Introduction}

It is important to measure the level of consciousness during general anesthesia to reduce the possibility of awareness, avoid overdoses, and improve patient outcomes. Monitoring is also essential for sedative titration to maintain adequate sedation. Therefore, several electroencephalography (EEG)-based devices are widely used to monitor the depth of anesthesia.

Recent reports have emphasized that the conscious state is associated with a rich repertoire of brain network activity and that brain functional connectivity patterns are diminished under anesthesia [1-3]. In functional magnetic resonance imaging

(c) This is an open-access article distributed under the terms of the Creative Commons Attribution Non-Commercial License (http://creativecommons.org/ licenses/by-nc/4.0/), which permits unrestricted non-commercial use, distribution, and reproduction in any medium, provided the original work is properly cited. 
studies, structured long-range connectivity remains in the unconscious state, whereas the rich repertoire of diverse functional configurations seen during consciousness is disrupted under general anesthesia [1]. Despite evidence that anesthesia alters the spatiotemporal dynamics of neural activity corresponding to the loss of consciousness, most current conventional monitoring systems are dependent on the amplitude of a single-channel electroencephalogram or spectral analysis (including biphasic analysis), thereby ignoring information related to dynamic connectivity [4]. A popular method used to infer connectivity among brain regions is phase synchronization with coherency or phase locking. This method requires stationary data with a rather long time series (several seconds) to reliably estimate the strength of functional connectivity $[5,6]$.

A phase-lag entropy (PLE) monitor (PLEM100; InBody Co., Korea) was recently introduced as a device to monitor the conscious state by measuring electroencephalographic signals and calculating PLE. Importantly, PLE is a new framework that predicts the complexity of communication by calculating the diverse connectivity of temporal patterns in phase relationships between two electroencephalographic signals from prefrontal and frontal montages, rather than the strength of phase coupling, as reflected in classical techniques. A high correlation has been reported between PLE and the level of consciousness [7].

This study was performed to investigate the ability of PLE to assess the level of sedation, as measured by the Observer's Assessment of Alertness/Sedation (OAA/S) scale, during propofol-induced sedation in patients undergoing upper limb surgery with a brachial plexus block. We simultaneously compared PLE with the bispectral index (BIS), the most commonly used parameter to monitor the level of consciousness.

\section{Materials and Methods}

\section{Patient population}

This study was approved by the Institutional Review Board of our hospital (Ref. 2017-05-018) and was registered with the International Clinical Trials Registry Platform (http://cris.nih. go.kr). Thirty patients (aged $\geq 18$ years, American Society of Anesthesiologists physical status I or II) scheduled for elective upper limb surgery under a brachial plexus block were recruited between July and September, 2017. Written informed consent was obtained from all patients on the day before the operation. Patients were excluded for the following reasons: the presence of neurological, hepatic, or renal disease; a history of obstructive sleep apnea; body mass index $\geq 30 \mathrm{~kg} / \mathrm{m}^{2}$; a history of adverse drug reactions to propofol or local anesthetics; a history of an allergic reaction to soybeans; or pregnancy. Patients who were on medications that affect the central nervous system (CNS) (e.g., anticonvulsants, hypnotics, and analgesics) were also excluded from the study.

\section{Study procedure}

When patients arrived at the operating room, noninvasive arterial blood pressure, electrocardiography, and peripheral oxygen saturation monitoring were initiated. An ultrasound-guided brachial plexus block was performed via the axillary approach. Next, $20 \mathrm{ml}$ of $0.45 \%$ ropivacaine were administered after negative aspiration and adequate sensory block was confirmed at 15 min after the injection of local anesthetics. In addition, CNS symptoms (e.g., feelings of lightheadedness and dizziness, visual and auditory disturbances, disorientation and occasional feelings of drowsiness, shivering, muscular twitching, tremors, and generalized convulsions of a tonic-clonic nature before propofol infusion) caused by the local anesthetics were observed and recorded. Disposable PLE and BIS sensors were placed on each patient's forehead, as recommended by the manufacturer, and connected to the PLE and BIS monitor (BIS ${ }^{\circledR}$ Monitor; Medtronic, Ireland), respectively. Baseline PLE and BIS values were recorded. The effect-site concentration $(\mathrm{Ce})$ of propofol was initially started at $0.5 \mu \mathrm{g} / \mathrm{ml}$ via a target-controlled infusion pump (Orchestra ${ }^{\circledR}$ Base Primea; Fresenius Vial, France) using Schnider's pharmacokinetic model [8]. During the study period, the level of sedation was assessed by one investigator using the OAA/S scale ( $5=$ responds readily to name spoken in normal tone; 4 = lethargic response to name spoken in normal tone, 3 $=$ responds only after name is called loudly or repeatedly, $2=$ responds only after mild prodding or shaking, $1=$ does not respond to mild prodding or shaking) [9].

The Ce was increased in increments of $0.2 \mu \mathrm{g} / \mathrm{ml}$ until an OAA/S score of 3 was reached, and then in increments of $0.1 \mu \mathrm{g} /$ $\mathrm{ml}$ until an OAA/S score of 1 was reached (loss of consciousness). Three minutes after the target $C e$ was reached, the PLE and BIS values, blood pressure, heart rate, and peripheral oxygen saturation were recorded, and the investigator evaluated the level of sedation. Oxygen was administered at $5 \mathrm{~L} / \mathrm{min}$ via a face mask until the end of the operation.

The primary goal of this study was to determine the correlation between the PLE values and the propofol-induced sedation level, as evaluated by the OAA/S scale, in comparison with the BIS values. Secondary goals were to compare PLE with the BIS value through prediction probability, and to examine the pharmacodynamic relationship between the sedation level and the Ce of propofol for each OAA/S score.

\section{Statistics}

Nonparametric Spearman correlation analysis was used to 
determine correlations between the OAA/S score and the PLE or BIS value. The PLE and BIS values were compared using the Wilcoxon matched-pairs signed-rank test or Student's $t$-test. Continuous variables are shown as means \pm standard deviations (SDs) or medians (25th-75th percentiles), while categorical variables are expressed as integers. All statistical analyses were performed using SigmaStat software, version 3.5 for Windows (Systat Software, Inc., USA). In all analyses, $\mathrm{P}<0.05$ was considered to indicate statistical significance.

$\mathrm{P}_{\mathrm{k}}$ values were calculated using Somers' d cross-tabulation statistic to evaluate the performance of the monitor [10]: $P_{k}=1$ indicates that the measure predicts the observed depth of anesthesia perfectly; $P_{k}=0.5$ indicates that the predictive accuracy of the measure is no better than chance $(50: 50)$; and $\mathrm{P}_{k}=0$ indicates that the measure has no predictive value.

\section{Probability of sedation and pharmacodynamic analysis}

The following sigmoid Emax model was used to determine the relationship between the probability of sedation $(P)$ and $C e$ of propofol:

$$
P(\text { sedation } \leq \mathrm{OAA} / \mathrm{S} \text { score of } i)=\frac{C_{e}^{r}}{C_{e 50_{-} O A A / S \text { score of } i}^{r}+C_{e}^{r}},
$$

where $C e_{50 \_O A A / S \text { score of } i}$ is the $C e$ associated with a $50 \%$ sedation probability $\leq$ the $i$ th OAA/S score, and $\gamma$ is the slope of the concentration versus the sedation probability curve. Model parameters were estimated using the option 'LIKELIHOOD LAPLACE METHOD $=$ conditional' in NONMEM ${ }^{\circledR} 7$ level 3 (ICON Development Solutions, Ireland). IIV of the $C e_{50}$ was fixed at zero.

\section{Results}

We collected 315 data points from 30 patients; the patients' characteristics are summarized in Table 1. There were no signs of CNS toxicity due to local anesthetics during the brachial plexus block. During propofol-induced sedation, no significant

Table 1. Patient Characteristics

\begin{tabular}{lc}
\multicolumn{1}{c}{ Variables } & Data values \\
\hline Age $(\mathrm{yr})$ & $53.6 \pm 14.8$ \\
Sex $(\mathrm{M} / \mathrm{F})$ & $15 / 15$ \\
Body mass index $\left(\mathrm{kg} / \mathrm{m}^{2}\right)$ & $23.8 \pm 2.7$ \\
Lean body mass $(\mathrm{kg})^{*}$ & $48.5 \pm 7.6$ \\
ASA physical status $(\mathrm{I} / \mathrm{II})$ & $19 / 11$
\end{tabular}

Values are presented as mean \pm SD or number of patients. ASA: American Society of Anesthesiologists. *Janmahasatian formula [22]. adverse events requiring cardiovascular or respiratory support occurred.

With increasing depth of sedation, the PLE and BIS values decreased progressively. As the OAA/S score decreased from 5 to 1 , the median values (25th-75th percentiles) of PLE and BIS decreased from 85 (79-89) to 59.5 (54-65) and from 88 (83-93) to 60.5 (54-63), respectively (Fig. 1). PLE was closely correlated with the OAA/S score that was similar to the correlation between the BIS value and OAA/S score during propofol-induced sedation (Spearman's Rho $=0.755$ and 0.788 , respectively; both $\mathrm{P}<0.001)$.

The $\mathrm{P}_{\mathrm{k}}$ values $(95 \% \mathrm{CI})$ of PLE and BIS were $0.731(0.708-$ $0.754)$ and $0.718(0.696-0.740)$, respectively.

The pharmacodynamic model parameters and logistic regres-

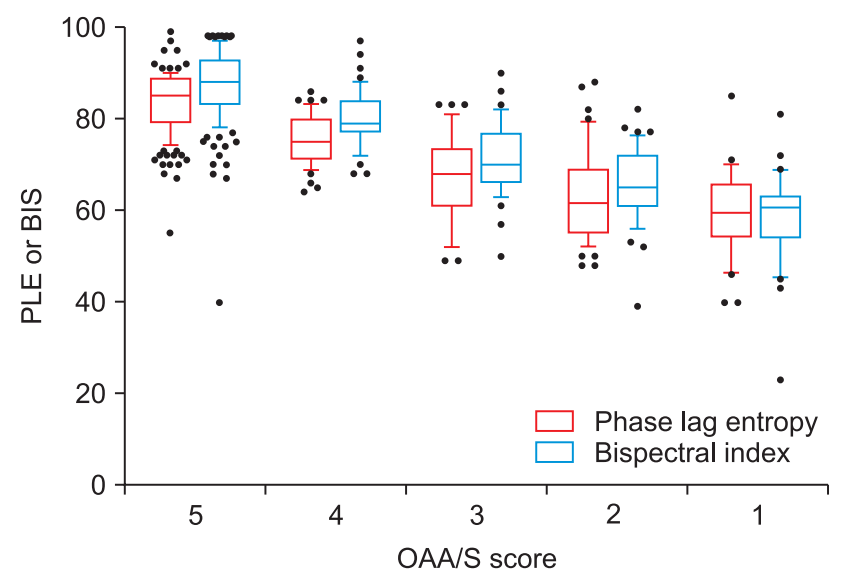

Fig. 1. Box and whiskers plot (10th, 25th, 50th, 75th, and 90th percentiles) of phase-lag entropy (PLE) or the bispectral index (BIS) at each Observer's Assessment of Alertness/Sedation (OAA/S) score. As the $\mathrm{OAA} / \mathrm{S}$ score decreased from 5 to 1 (i.e., $5 \rightarrow 4 \rightarrow 3 \rightarrow 2 \rightarrow 1$ ), the median values (25th-75th percentiles) of PLE and BIS gradually decreased from $85(79-89)$ to $75(71-79.8)$ to $68(61.8-74)$ to 61.5 (55-69) to 59.5 (54-65), and from $88(83-93)$ to $79(77-83.8)$ to 70 (66-77) to 65 (61-72) to 60.5 (54-63), respectively. The OAA/S score was closely correlated with PLE to an extent comparable with that of the BIS during propofol-induced sedation (Spearman's Rho $=0.755$ and 0.788 , respectively; $\mathrm{P}<0.001)$.

Table 2. Pharmacodynamic Model Parameters for Each OAA/S Score

\begin{tabular}{lc}
\hline Parameter & Estimate (\% RSE) \\
\hline$C e_{50 \_O A A / S \text { score of } 1}$ & $2.69(4.24)$ \\
$C e_{50 \_O A A / S \text { score of } 2}$ & $2.22(3.35)$ \\
$C e_{50 \_O A A / S}$ score of 3 & $1.96(3.27)$ \\
$C e_{50 \_O A A / S \text { score of } 4}$ & $1.67(2.76)$ \\
$\gamma$ & 9.56
\end{tabular}

OAA/S: Observer's Assessment of Alertness/Sedation, $\mathrm{Ce}_{50 \_ \text {OAA/S score of } n}$ : effect-site concentration associated with a $50 \%$ sedation probability $\leq$ the $i$ th OAA/S score, $\gamma$ : steepness of the concentration versus response relationship, RSE: relative standard error. 


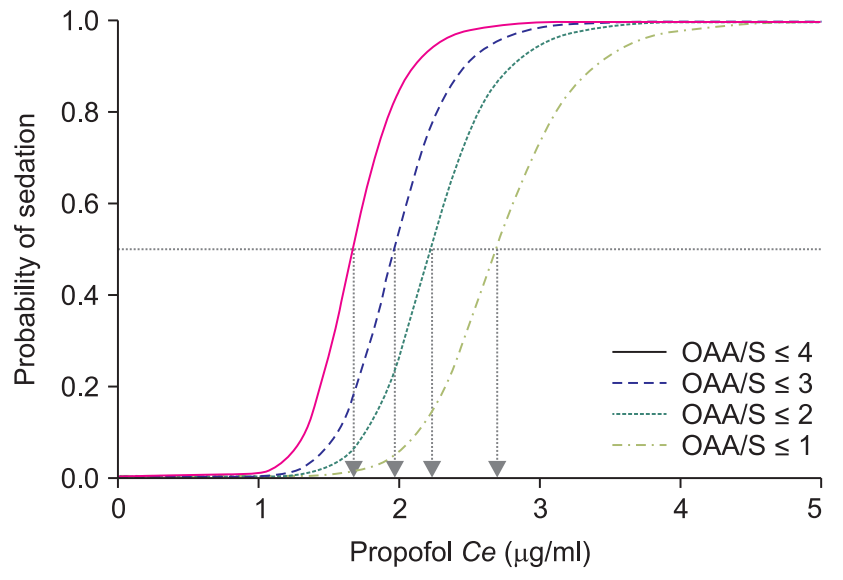

Fig. 2. Relationship between the probability of sedation and the Ce of propofol at each Observer's Assessment of Alertness/Sedation (OAA/S) score. The $C e_{50}$ (arrow) values were estimated to be 1.67, 1.96, 2.22, and $2.69 \mu \mathrm{g} / \mathrm{ml}$ for OAA/S scores of $\leq 4, \leq 3, \leq 2$, and $\leq 1$, respectively.

sion curve depicting the relationship between the Ce of propofol and the probability of sedation at each OAA/S score are shown in Table 2 and Fig. 2, respectively. The $C e_{50}$ values were estimated to be $1.67,1.96,2.22$, and $2.69 \mu \mathrm{g} / \mathrm{ml}$ for OAA/S scores of $\leq 4$, $\leq 3, \leq 2$, and $\leq 1$, respectively.

\section{Discussion}

This study demonstrates that PLE was closely correlated with the level of sedation measured by the OAA/S score. The ability of PLE to assess the depth of sedation was comparable with that of the BIS value. Recent studies suggest that the state of consciousness is more closely associated with the temporal dynamics of the functional network configuration among brain areas, rather than with the strength of static connectivity $[1,3,11]$. Lee et al. [7] revealed that the conscious state consists of diverse patterns of functional connectivity, whereas the anesthetized state has a few dominant patterns, suggesting stereotypic communication. However, current techniques for measuring connectivity in brain regions do not adequately explain the temporal dynamics of neurophysiological signals. Popular methods that use phase synchronization $[5,6]$ assume stationarity throughout the measurement period. The phase synchronization value is obtained by averaging phase differences across periods of several seconds, and important temporal information can be lost. Most EEGbased systems for monitoring the depth of anesthesia, including BIS, use this mechanism. Additionally, most such systems consider only the temporal characteristics of single-channel EEG, thereby disregarding spatial or connectivity information [4].

Unlike previous monitoring methods, PLE can be used to successfully evaluate the diversity of connectivity patterns, measured on time scales of tens of milliseconds, and to more accurately track the state of consciousness, compared with static strength-based connectivity. PLE quantifies the diversity of temporal patterns in the phase relationship between two signals and then incorporates the temporal dynamics of the instantaneous phase time series into the phase synchronization analysis. Therefore, PLE exhibits better performance when classifying states of consciousness, compared with a classical time-averaged connectivity method [12-14]. Anesthesia suppresses diverse connectivity patterns in brain activity [15-17]. The increased synchronization due to anesthesia is reinterpreted as a stereotypic communication and exhibits a substantial decrease in PLE, suggesting impairment in flexible corticocortical communication. Among several depth of anesthesia indicators studied recently, PLE exhibited the highest agreement with the level of consciousness [7]. The other indicators assessed only the temporal features of single-channel EEG and therefore dismissed spatial relationships across cortical areas; these relationships have critical relevance to the states of consciousness $[11,12]$.

In this study, PLE demonstrated adequate performance for the assessment of the depth of sedation, but it did not provide better performance than the BIS. We assumed that we could not detect differences in ability between PLE and BIS because these values and depth of sedation were evaluated $3 \mathrm{~min}$ after the target $C e$ of propofol was reached.

PLE comprises three subparameters: PLE $_{1}(8-32 \mathrm{~Hz}), \mathrm{PLE}_{2}$ (0.1-1 and 32-45 Hz), and BSR $(2-32 \mathrm{~Hz}) . \mathrm{PLE}_{1}$ and $\mathrm{PLE}_{2}$ are calculated in the alpha $(8-13 \mathrm{~Hz})$ and beta $(13-30 \mathrm{~Hz})$ bands for 4-s epoch data without overlap; slow-frequency $(0.1-1 \mathrm{~Hz})$ and gamma $(30-45 \mathrm{~Hz})$ bands are calculated for 8-s epoch data with $50 \%$ overlap. $\mathrm{PLE}_{1}$ reflects a light hypnotic state, whereas $\mathrm{PLE}_{2}$ reflects a surgical hypnotic state. BSR includes two types of burst-suppression detection: portions of isoelectric EEG and/ or a very low power frequency for $60 \mathrm{~s}$. PLE was calculated by combining $\mathrm{PLE}_{1}, \mathrm{PLE}_{2}$, and BSR with appropriate weights and was linearly scaled to the range of $0-100$.

In the present study, the relationship between the propofol $\mathrm{Ce}$ and depth of sedation was modeled. The difference between the $C e_{50}$ value of propofol for the probability of an $\mathrm{OAA} / \mathrm{S}$ score $\leq 4$ and that of an OAA/S score $\leq 1$ was only $1.02 \mu \mathrm{g} / \mathrm{ml}$, and the therapeutic index (TI) for sedation $\mathrm{TI}=\frac{\text { Ce50_OAA } / \mathrm{S} \text { score of } \leq 1}{\text { Ce50_OAA } / \mathrm{S} \text { score of } \leq 3}$ was 1.37 , indicating that the TI for the therapeutic range of sedation for propofol was very narrow. Propofol is widely used for sedation outside of the operating room, and many clinicians have expressed concern regarding its narrow therapeutic range that can lead to sudden deep sedation. Therefore, consciousness monitoring is required during propofol-induced sedation. Several studies have shown that 
monitoring using the BIS can be effective during propofol sedation in endoscopic and radiological interventions [18-20]. The results of the present study suggest that PLE can be applied fully to monitor the depth of consciousness during propofol sedation outside of the operating room. Here, propofol-induced sedation was evaluated only in healthy adult patients undergoing upper limb surgery under a brachial plexus block. As the plasma concentrations of local anesthetics increase, systemic toxicity may occur [21] and electroencephalographic signals may be affected, especially in relation to CNS toxicity. In the present study, no patients complained of symptoms of toxicity during the procedure; therefore, we can exclude the CNS effect of local anesthetics on monitoring. However, additional clinical studies are needed with other anesthetics, as well as larger and more heterogeneous populations, in order to determine the correlation between the OAA/S score and PLE and to investigate the accuracy of PLE.

In conclusion, we observed a good correlation between PLE and the depth of sedation, as measured by the OAA/S score, during propofol-induced sedation in patients undergoing upper limb surgery under a brachial plexus block. The monitoring performance of PLE was comparable with that of the BIS, indicating that it may be useful as a consciousness monitoring system for measuring sedation levels.

\section{Acknowledgments}

This study was supported by InBody Corporation and by the Industrial Strategic Technology Development program (N10047988, 2013). This research was made possible by permission from InBody to use the PLEM100 device and the PLEMES100 electrode.

\section{Funding Statement}

This study was funded by the Ministry of Industry and Trade.

\section{Conflicts of Interest}

No potential conflict of interest relevant to this article was reported.

\section{Author Contributions}

Mi Roung Jun (Data curation; Formal analysis; Investigation; Validation; Writing-original draft; Writing - review \& editing) Jae Hwa Yoo (Investigation; Validation)

Sun Young Park (Writing-review \& editing)

Sojin $\mathrm{Na}$ (Data curation)

Hyerim Kwon (Data curation)

Jae-Hwi Nho (Investigation)

Soon Im Kim (Conceptualization; Funding acquisition; Methodology; Supervision; Writing - original draft; Writing - review \& editing)

\section{ORCID}

Mi Roung Jun, https://orcid.org/0000-0003-0330-9102 Jae Hwa Yoo, https://orcid.org/0000-0003-0675-0761 Sun Young Park, https://orcid.org/0000-0003-2588-3324 Sojin Na, https://orcid.org/0000-0003-0240-3260 Hyerim Kwon, https://orcid.org/0000-0002-6241-4474 Jae-Hwi Nho, https://orcid.org/0000-0003-1053-456X Soon Im Kim, https://orcid.org/0000-0001-8884-4096

\section{References}

1. Hudetz AG, Liu X, Pillay S. Dynamic repertoire of intrinsic brain states is reduced in propofol-induced unconsciousness. Brain Connect 2015; 5: 10-22.

2. Sarasso S, Boly M, Napolitani M, Gosseries O, Charland-Verville V, Casarotto S, et al. Consciousness and complexity during unresponsiveness induced by propofol, xenon, and ketamine. Curr Biol 2015; 25: 3099-105.

3. Barttfeld P, Uhrig L, Sitt JD, Sigman M, Jarraya B, Dehaene S. Signature of consciousness in the dynamics of resting-state brain activity. Proc Natl Acad Sci U S A 2015; 112: 887-92.

4. Ferenets R, Vanluchene A, Lipping T, Heyse B, Struys MM. Behavior of entropy/complexity measures of the electroencephalogram during propofol-induced sedation: dose-dependent effects of remifentanil. Anesthesiology 2007; 106: 696-706.

5. Stam CJ, Nolte G, Daffertshofer A. Phase lag index: assessment of functional connectivity from multi channel EEG and MEG with diminished bias from common sources. Hum Brain Mapp 2007; 28: 1178-93.

6. Nolte G, Bai O, Wheaton L, Mari Z, Vorbach S, Hallett M. Identifying true brain interaction from EEG data using the imaginary part of coherency. Clin Neurophysiol 2004; 115: 2292-307.

7. Lee H, Noh GJ, Joo P, Choi BM, Silverstein BH, Kim M, et al. Diversity of functional connectivity patterns is reduced in propofol-induced unconsciousness. Hum Brain Mapp 2017; 38: 4980-95.

8. Schnider TW, Minto CF, Gambus PL, Andresen C, Goodale DB, Shafer SL, et al. The influence of method of administration and covariates on the pharmacokinetics of propofol in adult volunteers. Anesthesiology 1998; 88: 1170-82. 
9. Chernik DA, Gillings D, Laine H, Hendler J, Silver JM, Davidson AB, et al. Validity and reliability of the Observer's Assessment of Alertness/ Sedation Scale: study with intravenous midazolam. J Clin Psychopharmacol 1990; 10: 244-51.

10. Smith WD, Dutton RC, Smith NT. Measuring the performance of anesthetic depth indicators. Anesthesiology 1996; 84: 38-51.

11. Casali AG, Gosseries O, Rosanova M, Boly M, Sarasso S, Casali KR, et al. A theoretically based index of consciousness independent of sensory processing and behavior. Sci Transl Med 2013; 5: 198ra105.

12. Cimenser A, Purdon PL, Pierce ET, Walsh JL, Salazar-Gomez AF, Harrell PG, et al. Tracking brain states under general anesthesia by using global coherence analysis. Proc Natl Acad Sci U S A 2011; 108: 8832-7.

13. Lee H, Mashour GA, Noh GJ, Kim S, Lee U. Reconfiguration of network hub structure after propofol-induced unconsciousness. Anesthesiology 2013; 119: 1347-59.

14. Purdon PL, Pierce ET, Mukamel EA, Prerau MJ, Walsh JL, Wong KF, et al. Electroencephalogram signatures of loss and recovery of consciousness from propofol. Proc Natl Acad Sci U S A 2013; 110: E1142-51.

15. Bruhn J, Röpcke H, Hoeft A. Approximate entropy as an electroencephalographic measure of anesthetic drug effect during desflurane anesthesia. Anesthesiology 2000; 92: 715-26.

16. Bruhn J, Röpcke H, Rehberg B, Bouillon T, Hoeft A. Electroencephalogram approximate entropy correctly classifies the occurrence of burst suppression pattern as increasing anesthetic drug effect. Anesthesiology 2000; 93: 981-5.

17. Noh GJ, Kim KM, Jeong YB, Jeong SW, Yoon HS, Jeong SM, et al. Electroencephalographic approximate entropy changes in healthy volunteers during remifentanil infusion. Anesthesiology 2006; 104: 921-32.

18. Paspatis GA, Chainaki I, Manolaraki MM, Vardas E, Theodoropoulou A, Tribonias G, et al. Efficacy of bispectral index monitoring as an adjunct to propofol deep sedation for ERCP: a randomized controlled trial. Endoscopy 2009; 41: 1046-51.

19. Heuss LT, Schnieper P, Drewe J, Pflimlin E, Beglinger C. Conscious sedation with propofol in elderly patients: a prospective evaluation. Aliment Pharmacol Ther 2003; 17: 1493-501.

20. Dahaba AA, Lischnig U, Kronthaler R, Bornemann H, Georgiev V, Rehak PH, et al. Bispectral-index-guided versus clinically guided remifentanil/propofol analgesia/sedation for interventional radiological procedures: an observer-blinded randomized study. Anesth Analg 2006; 103: 378-84.

21. Neal JM, Woodward CM, Harrison TK. The American society of regional anesthesia and pain medicine checklist for managing local anesthetic systemic toxicity: 2017 version. Reg Anesth Pain Med 2018; 43: 150-3.

22. Janmahasatian S, Duffull SB, Ash S, Ward LC, Byrne NM, Green B. Quantification of lean bodyweight. Clin Pharmacokinet 2005; 44: 105165. 